\title{
Temporal and Spatial Pattern of Indole-3-acetic Acid Occurrence during Walnut Pistillate Flower Bud Differentiation as Revealed by Immunohistochemistry
}

\author{
Ying Gao, Hao Liu, Ningguang Dong, and Dong Pei ${ }^{1}$ \\ State Key Laboratory of Tree Genetics and Breeding, Research Institute of Forestry, Chinese \\ Academy of Forestry, Beijing 100091, P.R. China
}

\begin{abstract}
AdDitional INDEX wORDs. Juglans regia, Inflorescence, colloidal gold, immunohistochemical, auxin
ABSTRACT. We used anti-indole-3-acetic acid (IAA) monoclonal antibodies to monitor the temporal and spatial pattern of IAA during pistillate flower bud differentiation in the walnut (Juglans regia) cultivar Liaoning 1. Based on morphological changes, the process of pistillate flower bud differentiation was divided into five stages. The flower induction stage, which includes the early phase, midphase, and late phase, persisted from 25 Apr. to the end of May. The pedicel differentiation stage began on 5 June. The bract primordium stage began on 25 June and persisted through mid-March of the next year. Both the perianth and pistil differentiation stages persisted for nearly 2 weeks. During the floral induction period, little IAA was present in the shoot apical meristem (SAM); hence, the SAM may not always be a site of IAA production. IAA was obviously concentrated in cells of the first several layers of the SAM during pedicel primordium formation. High levels of IAA were also noted in the phyllome, young leaf tips, and vascular bundle of leaves and gemmae. This direct evidence indicates that no close relationship exists between IAA and physiological differentiation; instead, IAA may strongly affect morphogenesis. These findings comprise a first step toward elucidating the walnut flowering mechanism.
\end{abstract}

The plant hormone and signaling molecule auxin plays a key role during flower formation, organogenesis, and diverse physiological processes (Kamil et al., 2007; Valentina et al., 2008; Wu et al., 2008). The course of flower bud differentiation is crucial for processes of agriculture, horticulture, and plant breeding because flowering is the first step in sexual reproduction. Luckwill (1970) first demonstrated that auxin/cytokinin balance controls flower formation, and physiological and molecular studies on arabidopsis (Arabidopsis thaliana) have shown that IAA and auxin polar transport are almost certainly essential for flower formation (Aaron et al., 2003; Aloni et al., 2006; Li et al., 2009). The relationship between IAA and flower formation in perennial woody plants has been studied by highperformance liquid chromatography [HPLC (Guo et al., 2010; Li et al., 1996; Liu et al., 2007)], which indicated that high levels of IAA promote flower bud induction in apple trees (Malus pumila), whereas low levels promote flowering in loquat (Eriobotrya japonica) and chestnut (Castanea mollissima). Thus, the role of IAA is not uniform among plant species. Although few studies have investigated flower formation in walnut, Gao et al. (2010), Polito and Li (1985), and Xia and Xi (1989) examined walnut morphology and the period of flower bud differentiation. In addition, HPLC has been used to analyze endogenous auxin during flower bud differentiation in this tree species. Tong and Hao (1991) determined that IAA levels in the reproductive terminal bud are always low, especially marked during floral induction. Ferhad et al. (2010) found little difference in IAA levels between the two terminal shoot types, but the trends (increase first, then decrease, and increase finally)

Received for publication 24 Apr. 2012. Accepted for publication 16 July 2012. This work was supported by the National Natural Science Foundation of China (No. 31171933).

${ }^{1}$ Corresponding author. E-mail: peigu@caf.ac.cn. in IAA levels were similar to those reported by Tong and Hao (1991). IAA levels are related to walnut flower bud differentiation, but the pattern does not precisely reflect the distribution of auxin in tissues.

Determination of in situ auxin levels would represent an important step toward a better understanding of the biological significance of the hormone in developing tissues. Immunocytochemical techniques make practical use of differential combinations between antigens and antibodies. The methodology has evolved rapidly in biological and medical research and is a very important procedure for describing auxin distribution in plants (Dewitte and Van, 2001), facilitating the localization of auxins in situ. Monoclonal antibodies against IAA have been used in maize [Zea mays (Kerk and Feldman, 1995; Shi et al., 1993; Vysotskaya et al., 2007)], peanut [Arachis hypogaea (Moctezuma, 1999)], sunflower embryos [Helianthus annuus (Thomas et al., 2002)], arabidopsis tissues (Aloni et al., 2003; Orna et al., 2002), strawberry [Fragaria Xananassa (Hou and Huang, 2004, 2005)], and poplar 741 [Populus alba $\times$ $(P$.davidiana $\times P$. simonii $) \times P$. tomentosa (Dong et al., 2011)]. However, the distribution of IAA during walnut flower formation is unknown.

We describe the spatial distribution of endogenous IAA and its dynamics during walnut flower differentiation using an immunohistochemical approach. We provide a substantial contribution to understanding of the IAA mechanism at the cellular level during flower bud differentiation. This work will promote improved production and breeding of walnut.

\section{Materials and Methods}

Plant material. We used the protandrous precocious walnut cultivar Liaoning 1. Trees that were grown for 9 years 

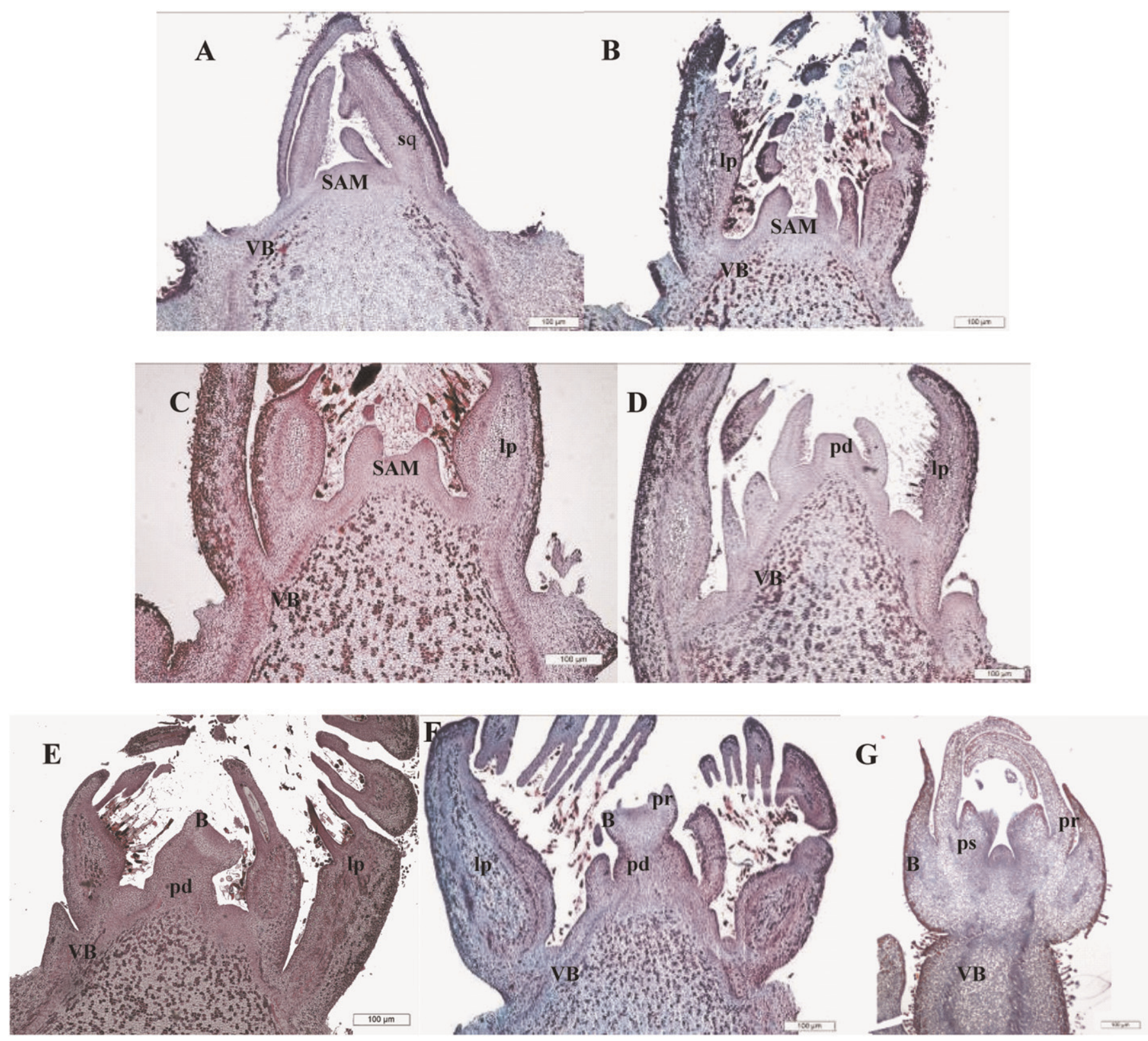

Fig. 1. Anatomy of walnut flower bud differentiation. (A-G) Longitudinal sections of flower buds; (A-C) floral induction periods; (D-G) pedicel (pd), bract (B), perianth (pr), and pistil (ps) differentiation stages; $\mathrm{SAM}=$ shoot apical meristem; sq = squama; $\mathrm{VB}=$ vascular bundle; lp = leaf primordial; $(\mathbf{A}-\mathbf{F})$ bar $=100 \mu \mathrm{m}$, (G) bar $=200 \mu \mathrm{m}$.

in Hebei province (lat. $39^{\circ} 26^{\prime} 31.40^{\prime \prime} \mathrm{N}$, long. $\left.115^{\circ} 31^{\prime} 53.72^{\prime \prime} \mathrm{E}\right)$ turned into steady reproductive growth and grew moderately. During flower bud differentiation (Apr. 2010 to Mar. 2011), we excised 20 terminal buds from the crown periphery of five trees every 3 or $5 \mathrm{~d}$, discarded the squama, and fixed specimens for anatomical observation and IAA immunohistochemical study.

ANatomical ObSERVATION OF FLOWER BUD DiFfERENTIATION. Excised samples were immediately fixed in freshly prepared $5 \%$ formaldehyde, $5 \%$ acetic acid, $70 \%$ ethanol for at least $24 \mathrm{~h}$, dehydrated through a series of ethanol steps $(70 \%, 85 \%, 95 \%$, $100 \%)$, cleared in dimethylbenzene, and embedded in paraffin. Vertical sections of $8 \mu \mathrm{m}$ thickness were cut with a rotary microtome (RM 2125RT; Leica, Wetzlar, Germany), transferred to glass slides, dried overnight, and stained with safranin-fastgreen (Sigma, St. Louis, MO). Photographs were taken on a microscope (BX41-12P02; Olympus, Tokyo, Japan). Three replicates were used for each sampling (Fig. 1).

INDOLE-3-ACETIC ACID IMMUNOHISTOCHEMICAL LOCALIZATION. Excised samples were immediately fixed in a $2 \%(\mathrm{w} / \mathrm{v})$ aqueous solution of 1-ethyl-3-(3-dimethylaminopropyl)carbodiimide [EDC (Sigma)] for $2 \mathrm{~h}$ under vacuum, post-fixed overnight in a solution containing $4 \%$ paraformaldehyde and $2.5 \%$ glutaraldehyde at $4{ }^{\circ} \mathrm{C}$, and rinsed in phosphate buffer $(0.2 \mathrm{M}$, $\mathrm{pH}$ 7.2) followed by dehydration in a graded ethanol series.

For immunohistochemical observation by light microscopy, samples were embedded in paraffin and sectioned to $8 \mu \mathrm{m}$ thickness (vertical sections and cross-sections). Sections were 

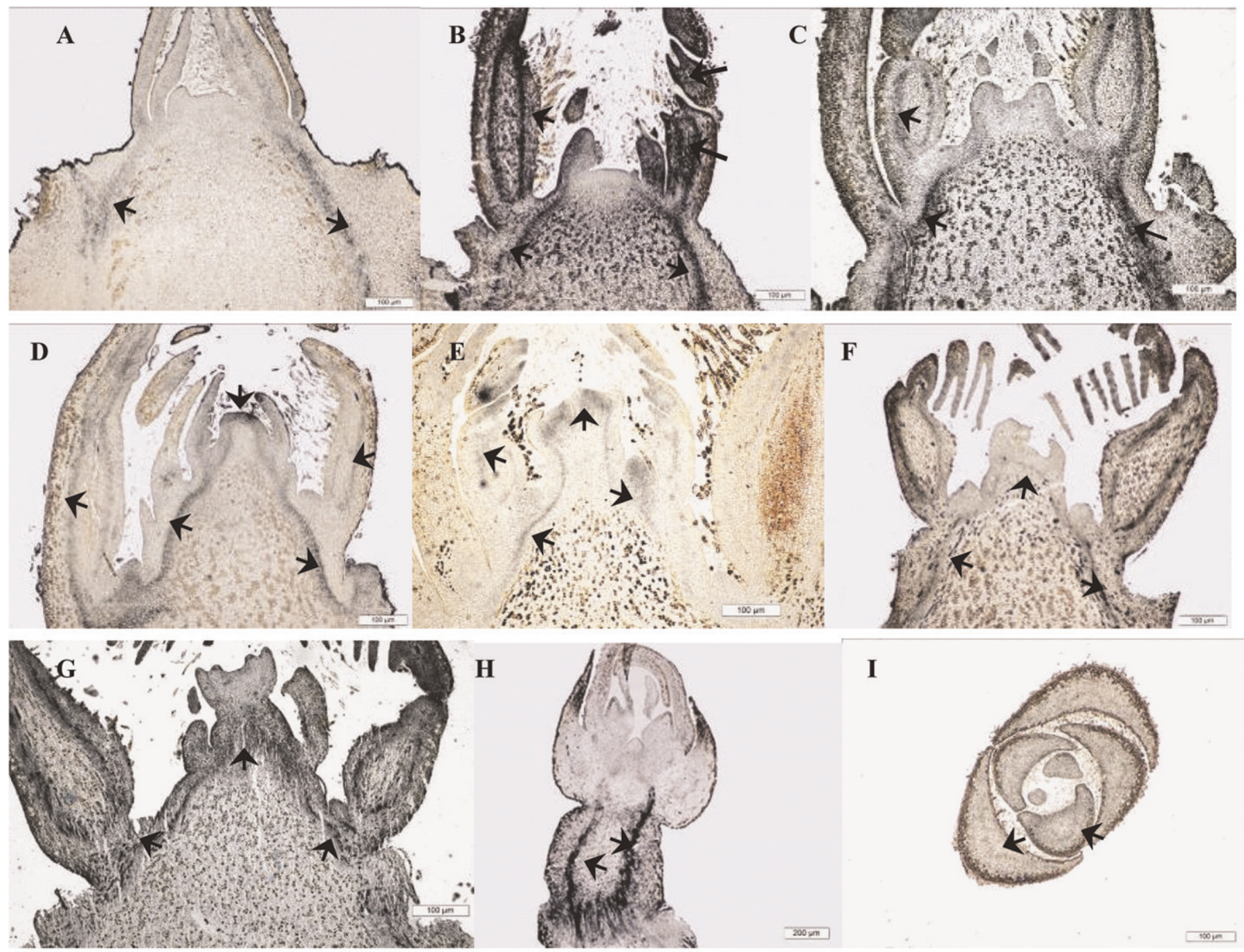

$\mathbf{H}$

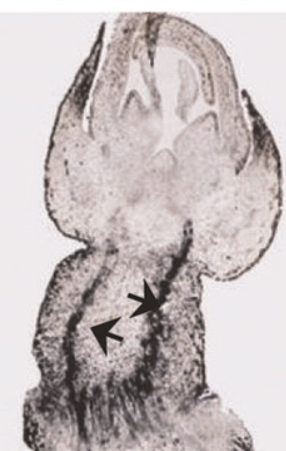

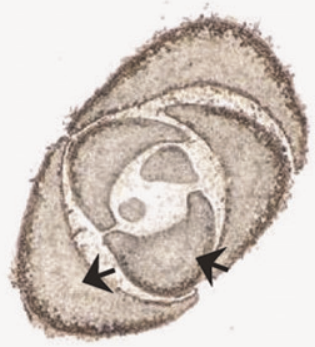

$\mathbf{J}$
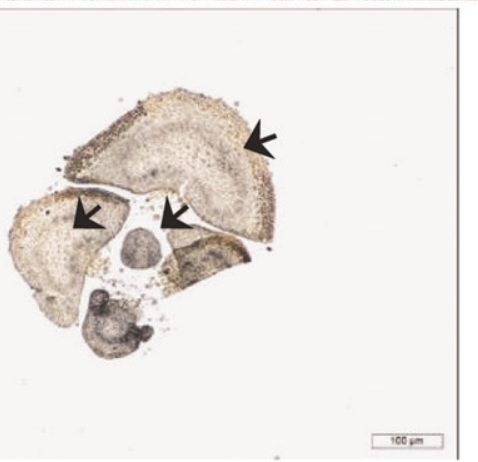

Fig. 2. Indole-3-acetic acid (IAA) immunolocalization in walnut flower bud tissues. (A-H) Longitudinal sections of differentiating flower buds; (A-C) floral induction; high levels IAA signals were observed around vascular bundles and tender squama in A (early phase); (B-C) midphase and late phase in floral induction; IAA is distributed mainly in the phyllome, young leaf, and vascular bundles and little occurs in the shoot apical meristem (SAM); (D) periods of pedicel differentiation; (E-F) periods of bract differentiation; (G) periods of perianth differentiation; (H) periods of pistil differentiation; at the onset of morphogenesis, IAA is concentrated in the SAM and in tissues undergoing differentiation; (I-J) cross-sections of flower bud; (I) early phase of floral induction; (J) midphase of floral induction; the IAA signal level in the apex increased during flower bud differentiation. The arrows indicate the locations of IAA. Bar $=100 \mu \mathrm{m}$ in all images except $\mathbf{H}$, in which the bar $=200 \mu \mathrm{m}$.

transferred onto glass slides. After overnight drying at $45^{\circ} \mathrm{C}$, sections were deparaffinized with xylene and hydrated in an ethanol/water series. The procedure for immunolocalization of IAA followed Holgate et al. (1983) with some modifications: slides were incubated for $30 \mathrm{~min}$ in a blocking solution $[0.05 \mathrm{M}$
Tris buffer pH 7.6 (TBS), 0.3\% (v/v) Triton X-100 (Sigma), $10 \%(\mathrm{v} / \mathrm{v})$ normal goat serum, $5 \%(\mathrm{w} / \mathrm{v})$ bovine serum albumin (BSA)] and incubated for $2 \mathrm{~h}$ at $37^{\circ} \mathrm{C}$ with primary IAA antibody (Agdia, Elkhart, IN) diluted 1:200 in TBS/BSA solution. Subsequently, sections were washed briefly in regular 

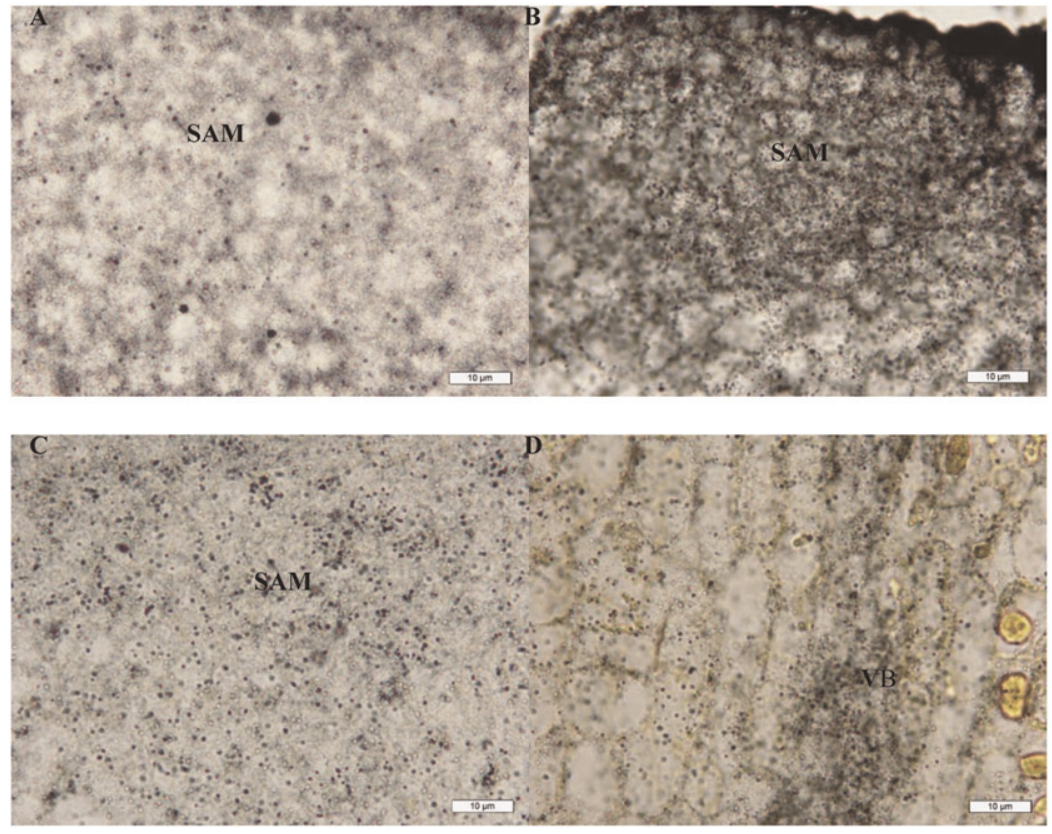

Fig. 3. Immunohistochemical localization of indole-3-acetic acid (IAA) in walnut flower bud during differentiation. The reddish brown silver particles were the IAA signal. (A) The distribution of IAA in shoot apical meristem (SAM) during floral induction (Fig. 2B); (B) the distribution of IAA in SAM during the pedicel stage (Fig. 2D); (C) the distribution of IAA in SAM during perianth period (Fig. 2G); (D) the distribution of IAA in young leaf. IAA was mainly distributed in the vascular bundles of young leaf. In the SAM, IAA increased when morphogenesis $(\mathbf{B})$; VB $=$ vascular bundles; bar $=10 \mu \mathrm{m}$.

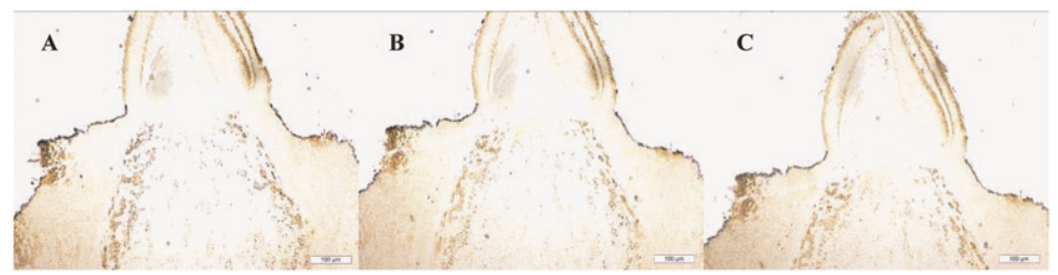

Fig. 4. Control of indole-3-acetic acid (IAA) immunolocalization in walnut flower bud tissues. (A-C) Controls with very low IAA signal levels; (A) no 1-ethyl-3-(3-dimethylaminopropyl)carbodiimide (EDC) prefixation; (B) no primary antibody; (C) no secondary antibody; bar $=100 \mu \mathrm{m}$. washing, the sections were submitted to the silver-enhancement reaction in a staining solution [0.1 M citrate buffer ( $\mathrm{pH} 3.5)$, $1.7 \%(\mathrm{w} / \mathrm{v})$ hydroquinone, $0.1 \%(\mathrm{w} / \mathrm{v})$ silver nitrate, $5 \%(\mathrm{w} / \mathrm{v})$ acacia]. As the color developed $(20 \mathrm{~min}$ ) on the sections, they were rinsed twice in water, dehydrated, mounted, observed, and photographed using a microscope (Olympus) (Fig. 2).

To verify the reliability of the immunolocalization technique and specificity of the anti-IAA antibodies, three negative controls were included. First, EDC was omitted during prefixation; second, the primary IAA antibody was omitted; and third, the secondary IAA antibody was omitted. All other procedures were performed as usual. Treatments were repeated three times.

INDOLE-3-ACETIC ACID SEMIQUANTITATIVE ANALYSIS. The distribution of IAA during flower bud differentiation in walnut flower bud was revealed by reddish brown silver particles in stained sections (such as Fig. 3A). For each tissue, 30 oil immersion light micrographs $(100 \times$ objective lens, $10 \times$ ocular lens) were taken at each stage of walnut pistillate flower bud differentiation. Using the image analysis software Image-Pro Plus6.0 (Media Cybernetics, Silver Spring, MD), we searched for silver particles, counted the number of unit areas, and then analyzed the distribution of IAA in different developing parts. The labeling density is presented as the number of silver particles per $100 \mu \mathrm{m}^{2}$. All treatments were repeated at least three times, and all samples were analyzed three times. Analysis of variance was performed, and significant differences between pairs of means were determined using a multiple-range test. Significance was assumed at $P<0.05$.

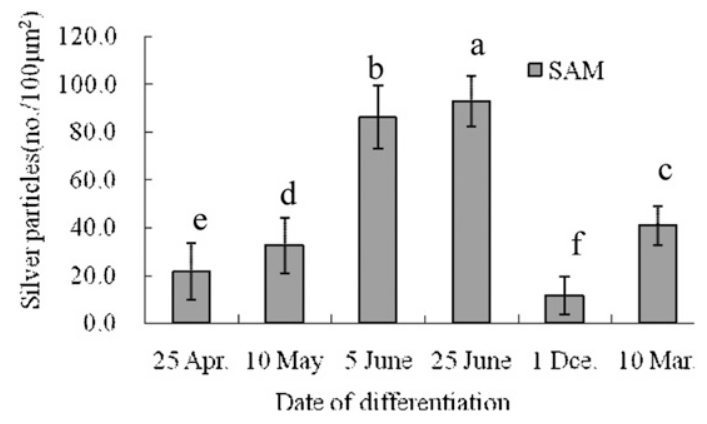

Fig. 5. Changes in indole-3-acetic acid (IAA) distribution during walnut flower bud differentiation of shoot apical meristem (SAM). Values are expressed as the mean number of silver particles per $100 \mu \mathrm{m}^{2} \pm$ SE. Significant differences through multiple-range test at $P<0.05$ indicated by a, b, c, d, e, and f.

salt rinse solution [0.05 M Tris buffer $\mathrm{pH} 7.6$ (TBS), $0.3 \%$ (v/v) Triton X-100, 5\% (w/v) BSA], blocked out again, and incubated for $1 \mathrm{~h}$ at $37^{\circ} \mathrm{C}$ with the gold-labeled goat antimouse $\operatorname{IgG}(10 \mathrm{~nm}$ diameter) diluted 1:50 in TBS/BSA solution. After

\section{Results}

Anatomy of flower bud differentiation. The whole process of flower bud differentiation lasted $\approx 1$ year across the 2 years. Five phases were distinguished based on anatomical structure. These were the floral induction period with three stages, the pedicel, bract, perianth, and pistil differentiation periods. Flower bud differentiation began on 25 Apr. 2010. In the initial stages, the apex was wrapped in several pea green squamas forming a semicircle. Subsequently the apex became even and elongated, and leaf primordia formed gradually over the following $15 \mathrm{~d}$ after early phase [10 May 2010 to 20 May 2010 (Fig. 1A-1C)]. During the period of morphological differentiation through 5 June 2010, each of the stages lasted $\approx 2$ weeks; changes were more marked thereafter. During the pedicel period, cells of apical meristem divided periclinally and pedicel primordia were erect (Fig. 1D). By 25 June 2010, the interspace of the SAM had hollowed and each side formed a protuberance, which was the bract primordium (Fig. 1E). This state persisted until the second year. During the perianth primordia period (10 Mar. 2011), two protuberances formed; 

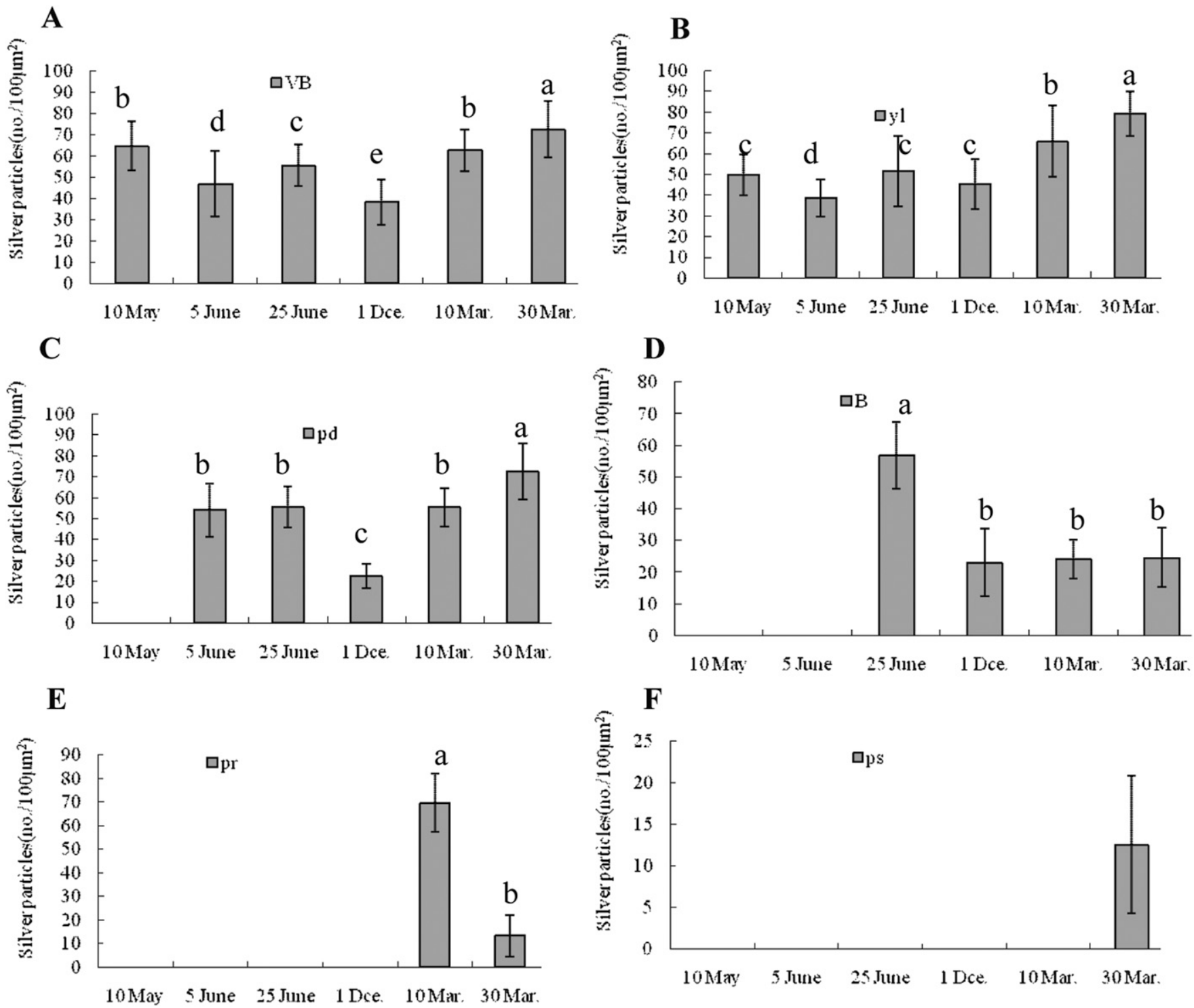

Fig. 6. Changes of density of silver particles in vascular bundle (VB). (A) Vascular bundle, (B) young leaf (yl), (C) pedicel (pd), (D) bract (b), (E) perianth (pr), (F) pistil (ps) of walnut flower bud during flower bud differentiation. Values are expressed as the mean number of silver particles per $100 \mu \mathrm{m}^{2} \pm \mathrm{SE}$. Significant differences through multiple-range test at $P<0.05$ indicated by a, b, c, d, and e.

these were perianth primordia (Fig. 1F). After 2 weeks, bracts and perianths were mature and the inside of the perianth was pistil-shaped [30 Mar. 2011 (Fig. 1G)].

INDOLE-3-ACETIC ACID IMMUNOHISTOCHEMICAL LOCALIZATION IN FLOWER BUD TISSUES. We examined the distribution of IAA through differentiation periods of the flower bud and leaf primordia using anti-IAA monoclonal antibodies (Fig. 2). In the early phase of floral induction, little IAA was present in the apex, but much larger quantities occurred in tender squama and vascular bundles (Figs. 2A and 2I). During flower bud differentiation, the level of IAA was higher in the apex than during the early phase but less than in leaf primordia and young leaves (Figs. 2B, 2C, 2J, 3A, and 3D). When morphogenesis began, IAA was concentrated in the first several layers of the SAM (Fig. 3B). IAA also occurred at high concentration in leaf primordia and vascular bundles (Fig. 2C). Little IAA was present during early bract differentiation. The highest level of IAA was in the SAM (under the first layer). The content of IAA decreased as trees moved into winter dormancy (Fig. 2F). The flower bud resumed differentiation in March of the second year. Perianth and pistil primordia formed in sequence. Vascular bundles contained high levels of IAA (Fig. 2G-H).

We verified the effectiveness of the immunolocalization technique and the specificity of the antibody with several control procedures. Because the monoclonal antibody was raised against free IAA crosslinked to BSA through its carboxyl group (Leverone et al., 1991), we tested unfixed tissues for the silver development reaction. No silver particles comparable to those in prefixed longitudinal flower bud sections (Fig. 4A) developed, indicating that prefixation with EDC is essential for free IAA detection by this antibody. When the primary (Fig. 4B) or secondary antibodies (Fig. 4C) were omitted, no signal was observed, suggesting that the silver development reaction is dependent on the presence of these antibodies in the tissue sections. 
Semiquantitative analysis of Indole-3-ACETIC ACID. Immunological and statistical data derived from analyses with Image-Pro Plus are depicted in Figures 5 and 6. The IAA signal was distributed widely among the SAM, young leaves, phyllomes, and vascular tissues, especially in splitting cells. The silver particles in the SAM stayed at a low level during the period of physiological differentiation and increased markedly when morphological differentiation began. With the onset of dormancy, the IAA signal decreased $(P<0.05)$. In the next year, the IAA signal strengthened as differentiation processes became accelerated (Figs. 3A-3C and 5). The number of silver particles in vascular tissues, young leaves, and pedicel decreased during dormancy. The trend in IAA distribution was decreased and then increased. We counted the numbers of silver particles in organs at different development stages and found that they were more concentrated in tissues undergoing differentiation. The numbers of silver particles in leaves and the vascular bundle keep high relatively (Fig. 6).

\section{Discussion}

WALNUT PISTILlate FlOWER FORMATION. Although the anatomical structure and differentiation processes of walnut pistillate flower formation have been studied for many years, we were able to make novel observations. Five differentiation periods were recognized in our study, and the floral induction period was divided into three stages by anatomical structure (Fig. 1). In other studies, only one stage was recognized during the physiological period (Li et al., 2011; Xia and Xi, 1989), whereas the midphase was a key process for the beginning of floral induction.

INDOLE-3-ACETIC ACID DISTRIBUTION IN THE WALNUT FLOWER BUD DURING FLOWER FORMATION. Auxin is a key hormone for flower formation. Most investigations of its effect on flowering have focused on quantitative analysis of IAA using diverse methods and on observations of flower characteristics after application of exogenous auxins or their inhibitors (Ferhad et al., 2010; Jia and Xiao, 2006; Tong and Hao, 1991). Studies on mutant arabidopsis have shown that disruption of auxin polar transport does not appear to prevent the transition from vegetative to reproductive growth, although it affects the formation of normal flower organs (Chen and Zhao, 2007; Gälweiler et al., 1998; Reinhardt et al., 2000). To date, the action of IAA in the walnut flower formation process has not been clearly elucidated. We showed that the IAA signal in walnut was distributed throughout the phyllome, young leaves, and vascular bundle tissues (Fig. 2) with little localization in the SAM during the flower induction period (Fig. 2A-C). As differentiation of the flower bud progressed, the IAA signal became concentrated in the SAM during the pedicel period (Fig. 2D). We suggest that IAA may not be correlated with the induction of flowers; instead, IAA accumulation in the SAM appears to induce morphogenesis, which is in agreement with studies on arabidopsis (Aloni et al., 2006). Although the results of our quantitative analysis (Fig. 5) are in accord with those of Tong and Hao (1991), we were better able to study the exact distribution of IAA. Dissecting out a few layers of cells for precise content analysis of SAM by HPLC [the procedure used by Tong and Hao (1991)] is exceedingly difficult because of contamination from other tissues such as the phyllome, young leaves, and vascular bundle tissues. In this study we selected 'Liaoning 1' for representation to investigate the distribution of
IAA during flower bud differentiation. The environment and cultivars may affect the starting time and speed of walnut flower bud differentiation; however, the process will not be changed (Li et al., 2011; Polito and Li, 1985; Zhang et al., 1995). Therefore, our study is universal.

Auxin transport may promote flowering, as shown by a study of the arabidopsis mutant pin-1 phenotype (Oka et al., 1999). The site of IAA production and its route of transport have also been investigated for several years. A general consensus holds that IAA, which plays a major role in regulating tissue differentiation and organogenesis, is produced in the SAM (Avery, 1935; Bartel, 1997). However, a conflicting view has been recently proposed. According to this new view, IAA is transported to the SAM rather than produced there (Orna et al., 2002). A component of this new view is the "IAA flow model," which is used to describe the temporal and spatial pattern of IAA flow in the shoot apex. In our study, little IAA was present in the SAM at the onset of floral induction, but concentrations were always relative high in the phyllome and vascular bundles (Fig. 2 A-C). We assume that IAA production may not always occur in the SAM or be influenced by consumption or export and that hormone detected there may have been transported from the phyllome, young leaves, or vascular bundles, where production occurs. These assumptions are tenuous and further study is required to determine the sites of IAA production and transportation in walnut.

\section{Literature Cited}

Aaron, M.R., P. Julie, S.W. Candace, and K.M. Gloria. 2003. Transport of the two natural auxins, indole-3-butyric acid and indole-3acetic acid, in Arabidopsis. Plant Physiol. 133:761-772.

Aloni, R., E. Aloni, M. Langhans, and C.I. Ullrich. 2006. Role of auxin in regulating Arabidopsis flower development. Planta 223: 315-328.

Aloni, R.K., M. Schwalm, and C.I. Langhans. 2003. Gradual shifts in sites of free-auxin production during leaf-primordium development and their role in vascular differentiation and leaf morphogenesis in Arabidopsis. Planta 216:841-853.

Avery, G.S. 1935. Differential distribution of phytohormone in the developing leaf of Nicotiana, and its relation to polarized growth. Bull. Torrey Bot. Club 62:313-330.

Bartel, B. 1997. Auxin biosynthesis. Annu. Rev. Plant Physiol. Plant Mol. Biol. 48:51-66.

Chen, Y.F. and D.Y. Zhao. 2007. A role for auxin in flower development. J. Integr. Plant Biol. 49:99-104.

Dewitte, W. and O.H. Van. 2001. Probing the distribution of plant hormones by immunocychemistry. Plant Growth Regulat. 33: $67-74$.

Dong, N.G., Y. Gao, W.L. Yin, and D. Pei. 2011. Immunogold silver localization of indole-3-acetic acid (IAA) during the rhizogenesis of in vitro poplar. Chinese Bul. Bot. 46:324-330.

Ferhad, M., B. Fikri, and B. Peyami. 2010. Endogenous hormone levels in bearing and non-bearing shoots of walnut (Juglans regia L.) and their mutual relationships. Acta Physiol. Plant. 32:53-57.

Gälweiler, L., C.H. Guan, A. Müller, E. Wisman, K. Mendgen, A. Yephremov, and K. Palme. 1998. Regulation of polar auxin transport by AtPIN1 in Arabidopsis vascular tissue. Science 282:22262230 .

Gao, Y., N.G. Dong, Z.H. Zhang, J.P Zhang, and D. Pei. 2010. Relationship between external morphology and anatomical structure of precocious walnut during pistillate flower bud differentiation. For. Res. 23:241-245.

Guo, Ch.Y., A.Z. Wei, P.H. Lu, and J.L. He. 2010. Changes of endogenous hormones during the younger shoot differentiation of chestnut. Acta Botanica Boreali-Occidentalia Sinica 30:2061-2066. 
Holgate, C.S., P. Jackson, and P.N. Cowen. 1983. Immunogold silver staining: New method of immunostaining with enhanced sensitivity. J. Histochem. Cytochem. 7:938-944.

Hou, Z.X. and W.D. Huang. 2004. Immunohistochemical localization of IAA in developing strawberry fruit. J. Hort. Sci. Biotechnol. 5:693-698.

Hou, Z.X. and W.D. Huang. 2005. Immunohistochemical localization of IAA and ABP1 in strawberry shoot apexes during floral induction. Planta 222:678-687.

Jia, R.F. and Q.W. Xiao. 2006. Analysis of applied effect of growth conditioners on walnut. J. Sichuan For. Sci. Technol. 27:77-79.

Kamil, R., K. Ljung, S. Vanneste, R. Podhorska, T. Beeckman, J. Friml, and E. Benkova. 2007. Ethylene regulates root growth through effects on auxin biosynthesis and transport-dependent auxin distribution. Plant Cell 19:2197-2212.

Kerk, N.M. and L.J. Feldman. 1995. A biochemical model for the initiation and maintenance of the quiescent center: Implications for organization of root meristems. Development 121:2825-2833.

Leverone, L.A., T.L. Stroup, and J.L. Caruso. 1991. Western blot analysis of cereal grain prolamins using an antibody to carboxyllinked indolacetic acid. Plant Physiol. 196:1076-1078.

Li, H.B., Y. Cheng, A. Murphy, G. Hagen, and T.J. Guilfoyle. 2009. Constitutive repression and activation of auxin signaling in Arabidopsis. Plant Physiol. 149:1277-1288.

Li, T.H., W.D. Huang, and Zh.Q. Meng. 1996. Study on the mechanisms of flower bud induction in apple tree. Acta Phytophysiol. Sinica 22:251-257.

Li, Y.T., Y.G. Zhao, K.Q. Yang, Y.Y. Fang, and L.Q. Hou. 2011. Studies on floral organ development process of precocious walnut (Juglans regia) by anatomia. Acta Hort. Sinica 38:434-440.

Liu, Z.L., Sh.Q. Lin, and H.B. Chen. 2007. Time course changes of endogenous hormone levels during the floral and vegetative buds formation in loquat (Eriobotrya japonica L.). Acta Hort. Sinica 34:339-344.

Luckwill, L.C. 1970. The control of growth and fruit fruitfulness of apple trees. trees, p. 237-254. In: Luckwill, L.C. and C.V. Cutting (eds.). Physiology of tree crops. Academic Press, New York, NY.

Moctezuma, E. 1999. Changes in auxin patterns in developing gynophores of the peanut plant (Arachis hypogaea L.). Ann. Bot. (Lond.) 83:235-242.
Oka, M., K. Miyamoto, K. Okada, and J. Ueda. 1999. Auxin polar transport and flower formation in Arabidopsis thaliana transformed with indoleacetamide hydrolase (iaaH) gene. Plant Cell Physiol. 40:231-237.

Orna, A.K., J.C. Cheng, L.J. Chen, E. Moctezuma, and Z.R. Sung. 2002. Indole acetic acid distribution coincides with vascular differentiation pattern during Arabidopsis leaf ontogeny. Plant Physiol. 130:199-209.

Polito, V.S. and N.Y. Li. 1985. Pistillate flower differentiation in english walnut (Juglans regia L.): A developmental basis for heterodichogamy. Sci. Hort. 26:333-338.

Reinhardt, D., T. Mendel, and C. Kuhlemeler. 2000. Auxin regulates the initiation and radial position of plant lateral organs. Plant Cell 12:507-518.

Shi, L., L. Miller, and R. Moore. 1993. Immunocytochemical localization of indole-3-acetic acid in primary roots of Zea mays. Plant Cell Environ. 16:967-973.

Thomas, C., R. Bronner, J. Molinier, E. Prinsen, H. van Onckelen, and G. Hahne. 2002. Immuno-cytochemical localization of indole-3acetic acid during induction of somatic embryogenesis in cultured sunflower embryos. Planta 215:577-583.

Tong, B.Q. and Z.Y. Hao. 1991. The pattern of endogenous hormones of pistillate flower differentiation in walnut (Juglans regia L.). Scientia Silvae Sinicae 27:401-409.

Valentina, C., M.A. Maria, F. Giuseppina, C. Paolo, and C. Maura. 2008. Auxin regulates Arabidopsis anther dehiscence, pollen maturation, and filament elongation. Plant Cell 20:1760-1774.

Vysotskaya, L.B., S.Yu. Veselov, and D.S. Veselov. 2007. Immunohistological localization and quantification of IAA in studies of root growth regulation. Russ. J. Plant Physiol. 54:827-832.

Wu, J.Z., Y. Qin, and J. Zhao. 2008. Pollen tube growth is affected by exogenous hormones and correlated with hormone changes in styles in Torenia fournieri L. Plant Growth Regulat. 55:137148.

Xia, X.Q. and R.T. Xi. 1989. The periods of physiological and morphological differentiation of pistillate flower buds in walnut (Juglans regia L.). J. Agr. Univ. Heibei 12:18-21.

Zhang, Z.H., W.J. Wang, Y. Gao, and G.Q. D. 1995. The process of flower differentiation on dichogamy of walnut. Acta Hort. Sinica 22:391-393. 